\title{
Enhancing the light-matter interaction using slow light: towards the concept of dense light
}

\author{
Luc Thévenaz*, Isabelle Dicaire, Sang-Hoon Chin \\ Ecole Polytechnique Fédérale Lausanne, STI-GR-SCI-LT Station 11, 1015 Lausanne, Switzerland
}

\begin{abstract}
A couple of experiments are here presented to clarify the impact of slow light on light-matter interaction. The experiments are designed, so that the process generating slow light and the probed light-matter interaction only present a marginal cross-effect. The impact of slow light on simple molecular absorption could be separately evaluated under either material or structural slow light propagation in the same medium and led to an entirely different response.
\end{abstract}

Keywords: Fiber optics, nonlinear fiber optics, slow and fast light, molecular absorption, stimulated Brillouin scattering.

\section{INTRODUCTION}

Slow light has attracted a lot of interest during the past decade, with the primary objective to realize all optical tunable delay lines for optical buffering ${ }^{1}$. The inherent limitation on the delay-bandwidth product to a value in the range of unity has seriously moderated the appeal of such systems for future telecommunication applications. The possibility to take full advantage of the real slowing of light and its consequent higher energy density for enhancing the light-matter interaction was then deeply questioned.

Recently, some research effort has been devoted to understanding the possibilities of slow light in enhancing light-matter interactions. For instance, the role of slow light in enhancing nonlinear effects has been theoretically investigated ${ }^{2,3}$. This enhancement is due to two contributions: the longer transient time of light pulses in optical media and the higher energy density due to spatial pulse compression. It has also been theoretically and experimentally proved that the extreme dispersion of slow light can lead to an enhancement of the spectral sensitivity of interferometers ${ }^{4}$. The role of slow light in gyroscopes has also been theoretically investigated ${ }^{5}$ and the possibility to use fiber Bragg gratings to realize ultrasensitive strain sensors has been experimentally proved ${ }^{6}$. Recent works ${ }^{7,8}$ have theoretically demonstrated that BeerLambert-Bouguer (BLB) absorption can be increased while light transmits through a special kind of microstructured photonic crystal (PC) cuvette. This absorption enhancement was attributed in these works ${ }^{7,8}$ to the slow group velocity in the cuvette. A simpler interpretation of these results would be that the PC structure acts as a quasi-cavity. The light bounces back and forth in the quasi-cavity several times, resulting in a longer interaction length with the liquid analyte.

In this paper, we present two conceptually simple experiments which were specifically designed to probe Beer-Lambert absorption under normal and slow light propagation, without modifying the experimental layout. The effect used for the generation of slow light does not impact on the fraction of the optical field in presence of the absorbing medium and was checked to keep the molecular absorption process in a non-saturation (linear) regime. This way the slow light generation does not modify the primary conditions in which light and molecules interact. Reversely, the experimental conditions were set, so that the molecular absorption does not decisively impact on the amount of slow light generation, or at least keeps it in a very minor fraction that can be easily and straightforwardly evaluated.

These two experiments are each realized to test a distinct manner to generate slow light: the first one to test material slow light (slow light in travelling-wave media) and the second one to test structural slow light (slow light in quasi-standing wave media, with multiple superposed fields). It will be shown that their impact on Beer-Lambert absorption is radically different. These demonstrations were experimentally carried out using a special kind of gas cell. A photonic crystal fiber (PCF) with solid core plays the role of the gas cell. The fiber holes are filled with gaseous acetylene at reduced pressure. A small fraction of the mode field (the evanescent field) propagates through the holes and thus experiences BeerLambert absorption. The fiber is made of a solid silica core, so that it can also be used as a Brillouin slow light medium to test material slow light. For testing structural slow light the fiber is simply placed in an all-fiber loop cavity, in which

*Luc.Thevenaz@EPFL:CH; phone +41 21693 4774; http://people.epfl.ch/luc.thevenaz

Advances in Slow and Fast Light V, edited by Selim M. Shahriar, Frank A. Narducci,

Proc. of SPIE Vol. 8273, 82731D · ( 2012 SPIE · CCC code: 0277-786X/12/\$18 · doi: 10.1117/12.914752 
the closed-loop factor can be easily varied. In both cases the absorption can be measured under normal and slow-light conditions without modifying the experimental layout. By comparing the relative absorption in the normal and slow light regimes, we can observe if slowing the light has any impact on the absorption by the gas.

\section{BEER-LAMBERT ABSORPTION MEDIUM}

The absorption of a gas analyte is usually expressed by means of the Beer-Lambert (BL) law. If light with an intensity $I_{\mathrm{o}}$ propagates through the gas cell, the transmitted intensity decays exponentially as $I=I_{0} \exp [-\alpha \cdot C \cdot L]$, where $C$ is the gas concentration, $L$ is optical path length and $\alpha$ is the molar absorption coefficient, which is a unique property of the gas, related to the imaginary part of the refractive index. Previous papers ${ }^{7}$ have suggested that the absorption coefficient is inversely proportional to the group velocity. In this paper we show experimentally that the relation between the effective absorption coefficient $\alpha$ and the group velocity is somehow dependent on the experimental conditions and lowing the light does not lead compulsorily to a change on the observed absorption. To clarify the relationship between BL absorption and slow light, a solid-core microstructured optical fiber replaces the classical gas cell. There are two main reasons for this: first, in such fiber, the optical mode is mainly confined to the solid core. Thus, the velocity of light propagating through the PCF can be controlled by manipulating the material properties of the core using a narrowband gain process such as stimulated Brillouin scattering. Second, with a proper choice of the fiber geometry, a non-negligible fraction of the guided light propagates through the holes filled with gas, and hence can probe Beer-Lambert absorption. The key advantage of this setup is that the effects of slow light and absorption are totally de-coupled, and hence we can vary one without having a direct impact on the other. Under these conditions, the equation of BLB absorption can be slightly modified as

$$
I=I_{o} \exp [-N \cdot \sigma \cdot f \cdot L]
$$

where $N$ is the gas molecular density, $\sigma$ is the absorption cross section, $f$ is the fraction of light in holes and $L$ is the optical length. Figure 1a shows the SEM image of the single-mode PCF filled with acetylene in the holes. Using the source-model technique (SMT) ${ }^{9}$, we calculated the mode field distribution along the core cross section of the PCF at a wavelength of $1535 \mathrm{~nm}$, as shown in Figure $1 \mathrm{~b}$. The evanescent part of the guided field propagating in the gas, representing $2.9 \%$ of the total power, is shown in Figure 1c.
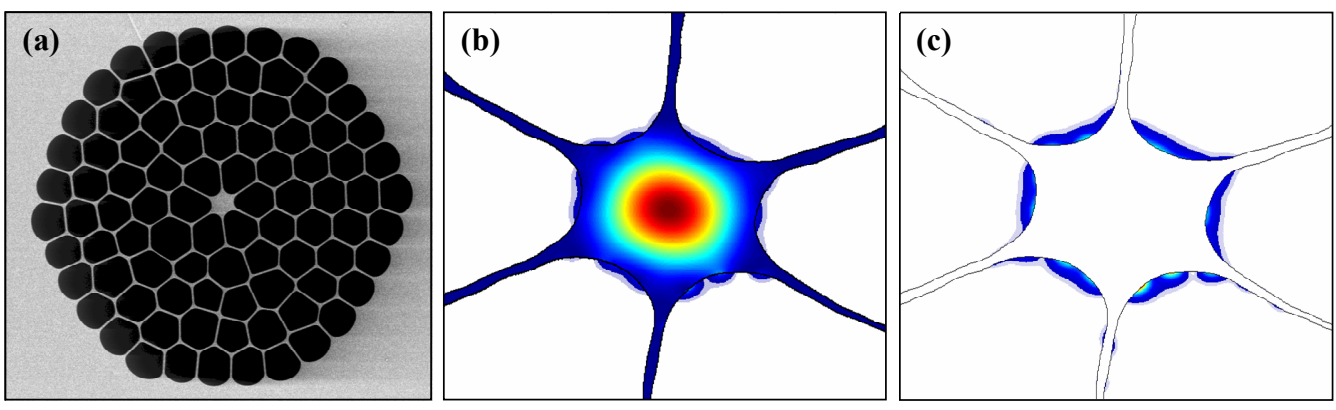

Figure 1: (a) SEM image of the solid-core microstructured fiber. (b) Calculated mode field distribution of the fundamental mode and (c) the small evanescent fraction of the guided field present in air holes.

\section{EXPERIMENTAL TEST USING MATERIAL SLOW LIGHT}

In this first experiment ${ }^{10}$ the property that the entire wave front of a guided optical mode propagates at the same velocity is used. The light in the guided mode is slowed in the solid silica fiber core using stimulated Brillouin scattering ${ }^{11}$ while the molecular absorption is probed by the evanescent field of the same guided mode.

As a Brillouin gain medium, a $9.18 \mathrm{~m}$-long PCF was used. The Brillouin spectrum of this fiber was characterized, showing a Brillouin shift of $10.85 \mathrm{GHz}$ and an SBS gain bandwidth of $38 \mathrm{MHz}$. A block diagram of the implementation of the experiment is shown in Fig. 2. A commercial distributed feedback (DFB) laser diode, operating at $1535 \mathrm{~nm}$, was used as the light source and its output was split using a directional coupler. One branch was strongly boosted using a 
high power erbium-doped fiber amplifier (EDFA) with $30 \mathrm{dBm}$ saturation power so as to play the role of the Brillouin pump. The output of the EDFA was precisely controlled by a variable optical attenuator before entering into the gas cell. The other branch was modulated through an electro-optic Mach-Zehnder intensity modulator (EOM) at the Brillouin frequency shift so as to generate two first-order sidebands in a suppressed carrier configuration. The lower-frequency sideband was then filtered by a fiber Bragg grating (FBG) and launched into the fiber as the signal frequency.

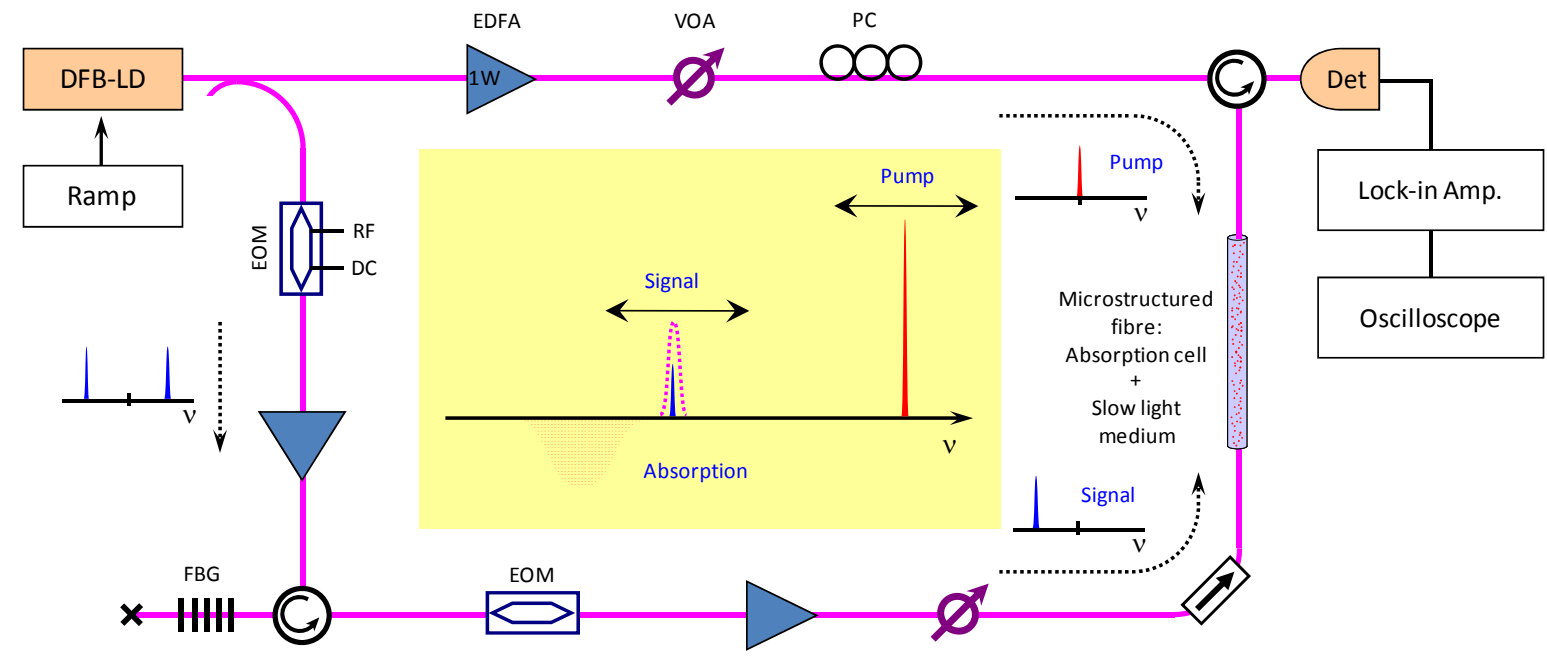

Figure 2: Experimental set-up simultaneously realizing a slow light element and a gas absorption cell in the same microstructured fibre. The optical frequency of the signal amplified and slowed down by stimulated Brillouin scattering can be scanned through an acetylene absorption line. The experimental arrangement makes the frequency separation between pump and signal constant during the frequency scan.

We first measured the amount of group velocity change as a function of the pump power. For this measurement, the frequencies of the signal and pump waves were spectrally placed in a region where absorption resonances are absent. To determine precisely the SBS-induced group delay, the signal wave was sinusoidally modulated at $1 \mathrm{MHz}$ by another external EOM. The phase of the sine wave after propagation through the PCF was measured while the pump power was incremented by $100 \mathrm{~mW}$ steps from 0 to $600 \mathrm{~mW}$. This way the group delay change achieved through the PCF for different pump powers was accurately determined from the differential phase shift. Additionally, the transit time through the fiber was also determined by making the same measurement with and without the PCF. The group velocity change is quantified by defining a slow-down factor $S$ corresponding to the ratio between the transit times through the gas cell with and without the pump, respectively. The largest time delay achieved through the PCF was $11.7 \mathrm{~ns}$, corresponding to a $26 \%$ reduction of the group velocity, at a pump power of $600 \mathrm{~mW}$.

To observe an absorption resonance of acetylene, the frequency of the signal was swept all over the absorption line, simply by introducing a slow variation of the current applied to the initial DFB laser. Since the signal and pump waves were generated from the same DFB laser source, the pump frequency was also swept perfectly synchronously, preserving the exact spectral distance between signal and pump that matches the Brillouin shift. Consequently, the pump constantly generated a Brillouin gain resonance centered at the signal frequency, even though the signal frequency was swept across the absorption line. The intensity of the signal wave was also sinusoidally modulated at $100 \mathrm{kHz}$ by an EOM to obtain a clean signal using a lock-in detection. The signal wave was then amplified using another EDFA, so as to even further improve the signal-to-noise ratio, still avoiding saturation of the Brillouin amplifier. The output power was precisely adjusted before entering into the gas cell using a VOA in order to avoid any risk of saturation of the atomic transition ${ }^{12}$. The signal amplitudes after propagating through the fiber gas cell were recorded for different pump powers on a digital oscilloscope while the laser frequency was swept.

Fig. 3a shows the measured normalized absorption when the signal is centered in the $\mathrm{P}(17)$ absorption line at $1535.453 \mathrm{~nm}$. The pump power was incremented following the same sequence as during the group delay calibration. Therefore, the curves are fully representative of the gas absorption while the signal propagates through the gas cell at different group velocities. The absorption curves were normalized in logarithmic scale for a better comparison of the absorption levels. To evaluate the confidence level, measurements were repeated 5 times for each power levels, so that 
the mean value and the standard deviation of the peak attenuation due to the BL absorption could be accurately estimated. These values representing the absorption at the center of the molecular line are plotted as a function of the slowdown factor in Fig. 3b. It is clearly observed that BL absorption is totally independent on the slow-down factor $S$, hence the group velocity. The same experiment was also performed for the $\mathrm{P}(15)$ and $\mathrm{P}(19)$ absorption lines at $1534 \mathrm{~nm}$ and $1536 \mathrm{~nm}$, respectively, resulting in very similar results despite fairly different magnitudes for their absorption coefficient $\alpha$.
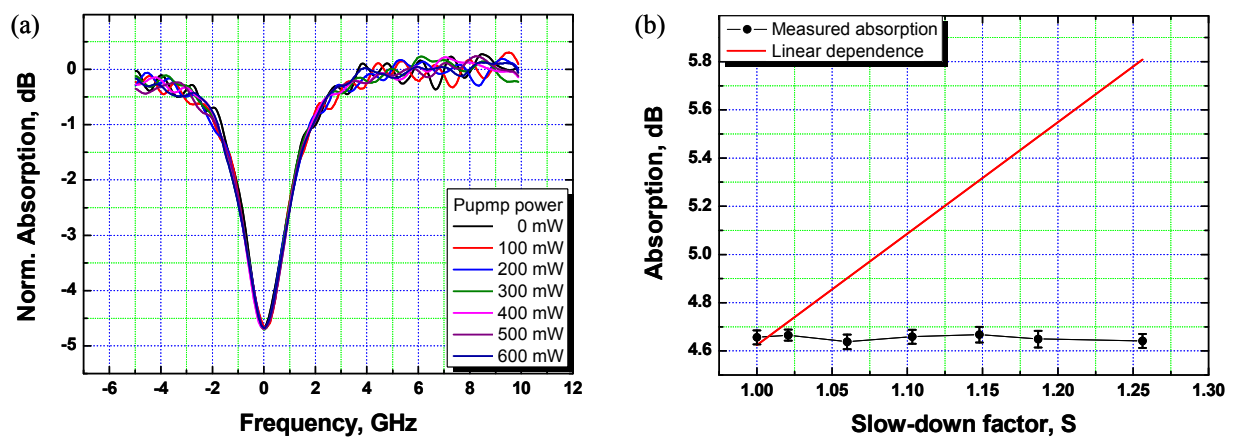

Figure 3: (a) Variation of the signal amplitude when performing a spectral scan through the acetylene P17 line $(\lambda=1535.40 \mathrm{~nm})$ after propagation through the solid-core PCF (in logarithmic scale for different pump powers). (b) Measured optical power loss at the peak attenuation due to the Beer-Lambert absorption as a function of the slowdown factor. The error bars show the measured standard deviation on the attenuation measurement and the red line represents the hypothetical response expected for an absorption coefficient inversely proportional to the group velocity.

It could be argued that the slow light induced delay ( $\sim 10 \mathrm{~ns})$ is negligible compared to the signal period (10 $\mu$ s) of the sine wave used for the measurements and thus does not expand substantially the interaction time to lead to an observable effect. To decisively clarify this issue, we repeated this experiment using a 22 ns FWHM optical pulse as signal wave, shorter than the $45 \mathrm{~ns}$ propagation time through the gas fiber cell. This way the time spent by the light pulse in the gas cell is clearly and noticeably modified by slow light. It should be nevertheless clarified that the duration of the light pulse is independent of the group velocity and therefore slowing the light does logically not modify the interaction time, as it is often erroneously claimed. This experiment resulted in the same flat response, confirming that BL absorption is not influenced by pure group velocity changes.

It was also checked that the fast light effect due to the acetylene molecular absorption line is negligible. A simple calculation shows that it is 140 times smaller than the largest slowing induced by Brillouin scattering. In all cases this minor fast light effect is identical in all Brillouin pumping situations and is thus similar in normal and slow light situations.

\section{EXPERIMENTAL TEST USING STRUCTURAL SLOW LIGHT}

In cavity ring resonators substantial group delays can be obtained for light signals matching the resonator frequency and can be attributed to light recirculating in the resonator. Cavity ring resonators essentially behave as frequency filters because wavelengths fitting an integer number of times within the cavity optical length will be selected and the other wavelengths will be rejected. Strong waveguide dispersion effects can therefore be obtained in such devices. A macroscopic version of microring resonators was built using fibre optics to provide a series of closely packed resonances inside a given gas-phase optical absorption resonance. The experiment is designed so that the molecular absorption and the slow light generation are negligibly correlated and can be controlled independently, as it could be done with the material slow light experiment. The macroscopic cavity can be conveniently opened or closed to operate either in standard or in slow-light regime, leaving the absorbing fibre segment untouched ${ }^{13}$. Closing the cavity results in delayed propagation and longer effective optical path lengths according to the cavity round-trip loss. The theoretical group delay is thus only function of the recirculation factor $\eta(0<\eta<1)$ for long cavity ring resonators:

$$
\tau_{D}=\frac{\eta}{1-\eta} \tau
$$

where $\tau$ is the cavity round-trip time. 
The experimental layout is depicted in Fig. 4, implementing a long fibre cavity ring resonator system. Light from a DFB laser diode operating at $1534 \mathrm{~nm}$ was launched into the fibre cavity. The optical power of the light signal was precisely controlled using a variable optical attenuator (VOA) before entering the cavity as to avoid saturation of the erbiumdoped fibre amplifier (EDFA) placed inside the cavity. The EDFA provided gain to the light signal to compensate any loss present in the cavity. A fibre Bragg grating (FBG) operated in reflection was used to filter out the amplified spontaneous emission coming from the EDFA and a second VOA was used to continuously vary the cavity round-trip loss. To determine precisely its value, cavity ring down measurements were performed by sending short optical pulses into the cavity and detecting the pulse intensity decaying with each cavity round-trip. For these measurements the laser frequency was positioned in a spectral region where absorption resonances were absent and the pulses were generated using an electro-optic Mach-Zehnder intensity modulator (EOM).

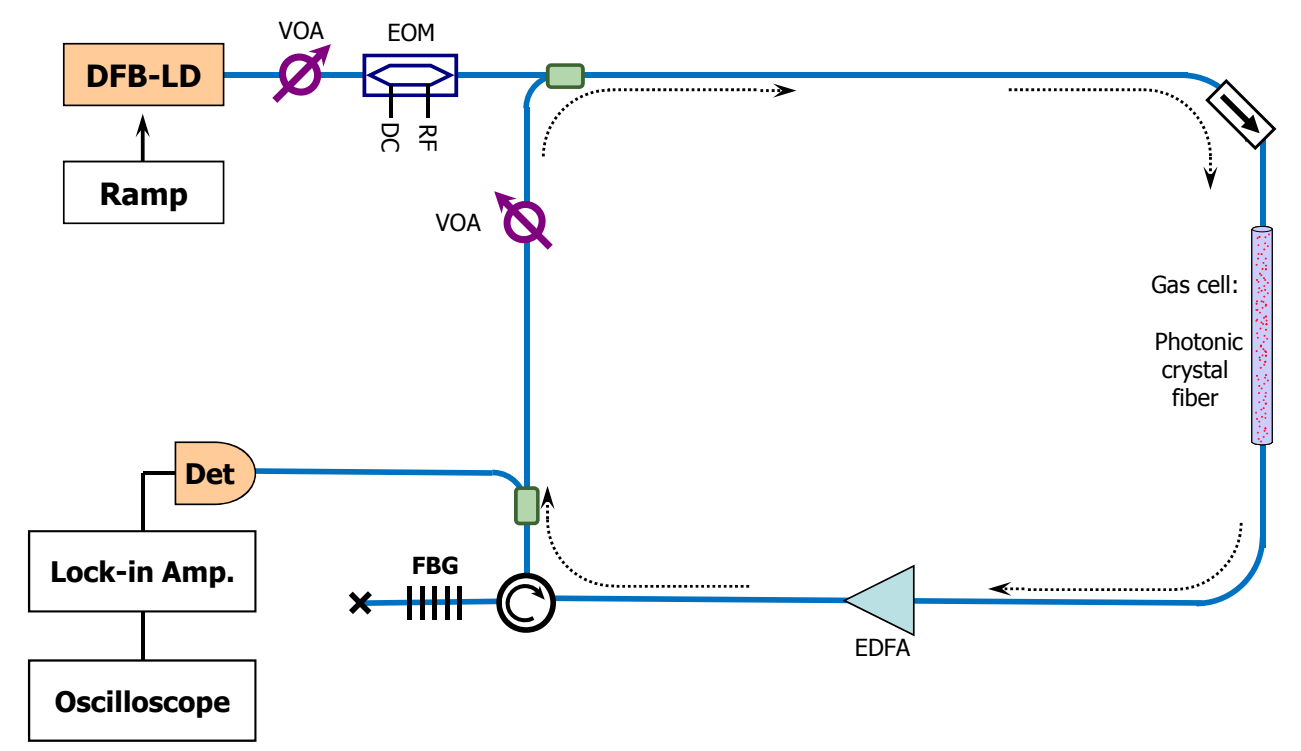

Figure 4. Experimental setup realizing structural slow light using a long fiber ring resonator system. Variable group delay is achieved by modifying the propagation loss in the fibre loop.

The cavity-induced group delay was then controlled by continuously varying the round-trip loss using a VOA. Semiquantitative measurements of the group delay were performed by using $3.8 \mu$ s optical pulses. Relatively long optical pulses should be used so that they could be much narrower in the frequency domain than the free spectral range of the cavity. The cavity acting as a frequency filter, the pulse width should be as narrow as possible in the frequency domain to avoid any frequency filtering effect, which would lead to temporal broadening. However the pulse width in the time domain cannot be increased arbitrarily as it will decrease the precision of the measured group delay. The value of $3.8 \mu \mathrm{s}$ is therefore a compromise between avoiding pulse temporal broadening and distortion and obtaining a relatively precise measurement of the pulse delay. The pulse delay measurements are shown in the left panel of Fig. 5 for several roundtrip losses.

To determine precisely the cavity-induced group delay, the output of the laser diode was sinusoidally modulated at $70 \mathrm{kHz}$ and $95 \mathrm{kHz}$ by an electro-optic Mach-Zehnder intensity modulator (EOM) and the phase of the sine wave was measured after propagation through the cavity using a lock-in amplifier. Subtracting this value from the phase of the sine wave measured with the cavity opened gave the phase shift value from which the group delay could be obtained. The phase shift measurements were performed at two different modulation frequencies to verify the accuracy of the measured group delay. The largest time delay achieved through the cavity was $\sim 700 \mathrm{~ns}$ for a loop time of $260 \mathrm{~ns}$, corresponding to a group velocity reduced to $27 \%$ of its nominal value. The phase shift measurements are shown in the right panel of Fig. 5 as a function of the cavity round-trip loss with the dashed line representing the theoretical group delay (Eq. 2). 

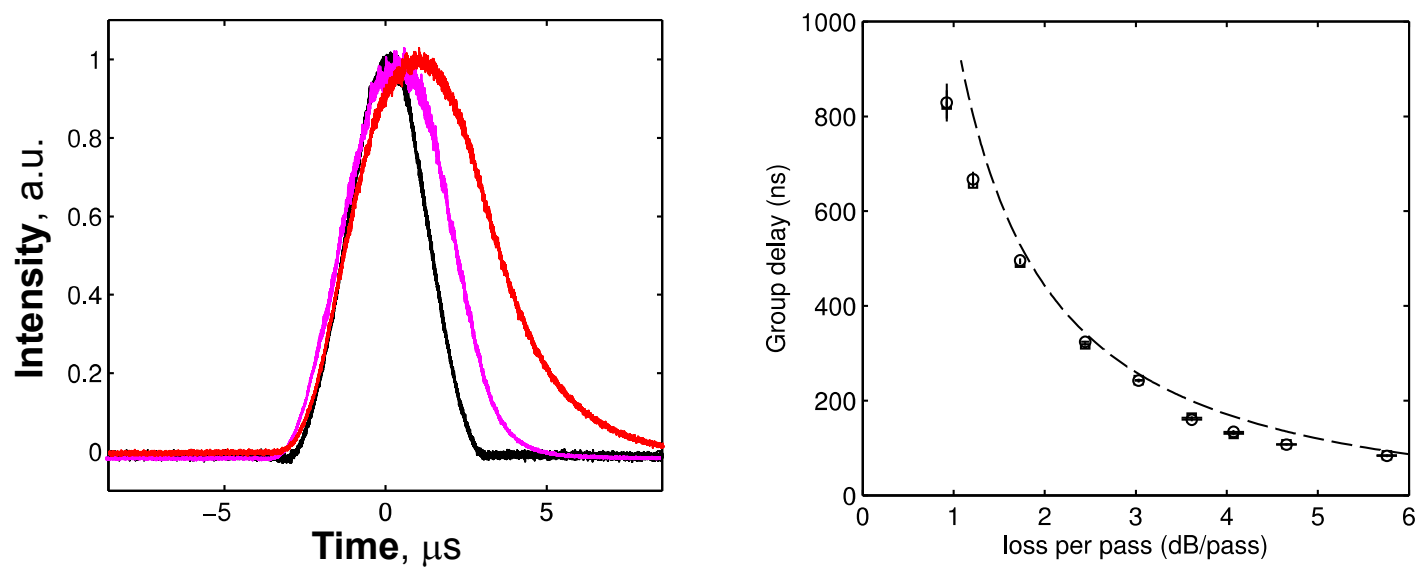

Figure 5. Left: Pulse delay measurements shown for several cavity round-trip losses. Decreasing the cavity loss leads to longer pulse arrival time through light recirculation. Right: Group delay measured as a function of the cavity round-trip loss using the phase shift technique. The dashed curve shows theoretical dependence obtained from Eq. 2 for a cavity round-trip time of $260 \mathrm{~ns}$.

Absorption profiles were obtained by applying a voltage ramp signal to the laser diode combined with lock-in detection to acquire clean signals. Figure 6 shows absorption profiles for the P17 acetylene line in the solid core PCF gas cell for several group indices and experimentally demonstrates substantial enhancement of the molecular absorption effect. Experimental data points were fitted with a theoretical model we developed and show good agreement with the expected absorption enhancement. The slight nonlinear dependence on the group index simply results from the change induced by the molecular absorption in the cavity round-trip loss; this effect is thus included in the model. This structural slow-light experiment clearly demonstrates the enhancement of the molecular absorption effect as a function of the cavity-induced group delay. This experimental result can actually be explained out of the framework of slow light theory, by looking at the actual intensity building up inside the cavity.
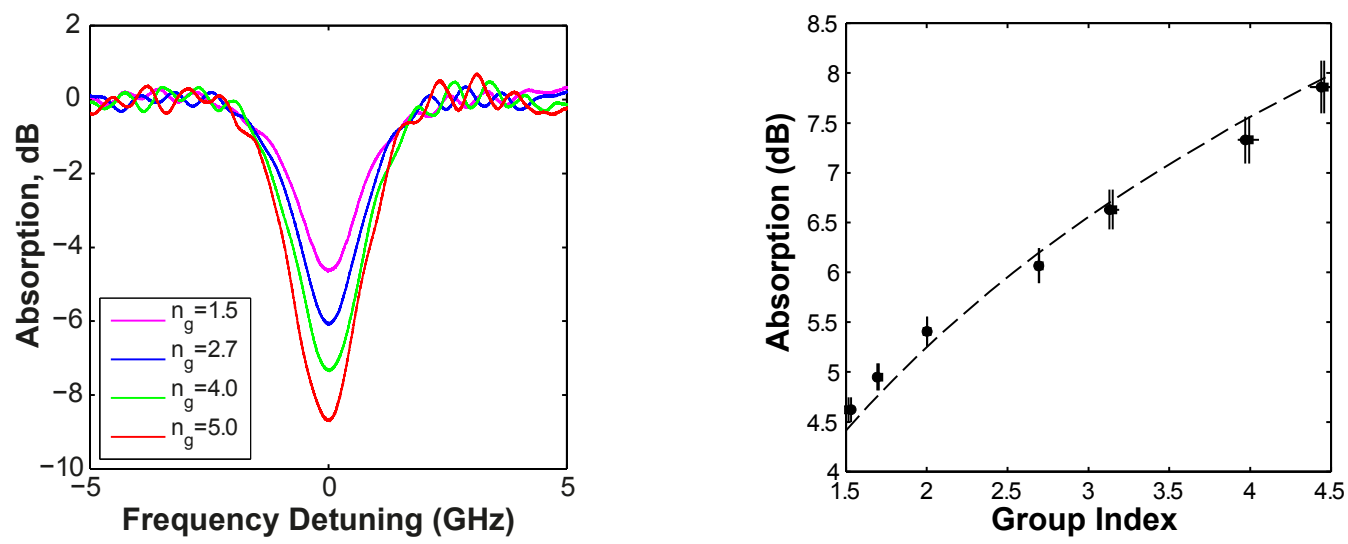

Figure 6. Left: Absorption profiles measured in the solid-core PCF for the P17 line $(\lambda=1535.40 \mathrm{~nm})$ for several group indices. Right: Absorption enhancement as a function of the group index. The dashed line represents the absorption predicted by our model.

This structural slow-light experiment clearly demonstrates the enhancement of the molecular absorption effect as a function of the cavity-induced group delay. This experimental result can actually be explained out of the framework of slow light theory, by looking at the actual intensity building up inside the cavity. 


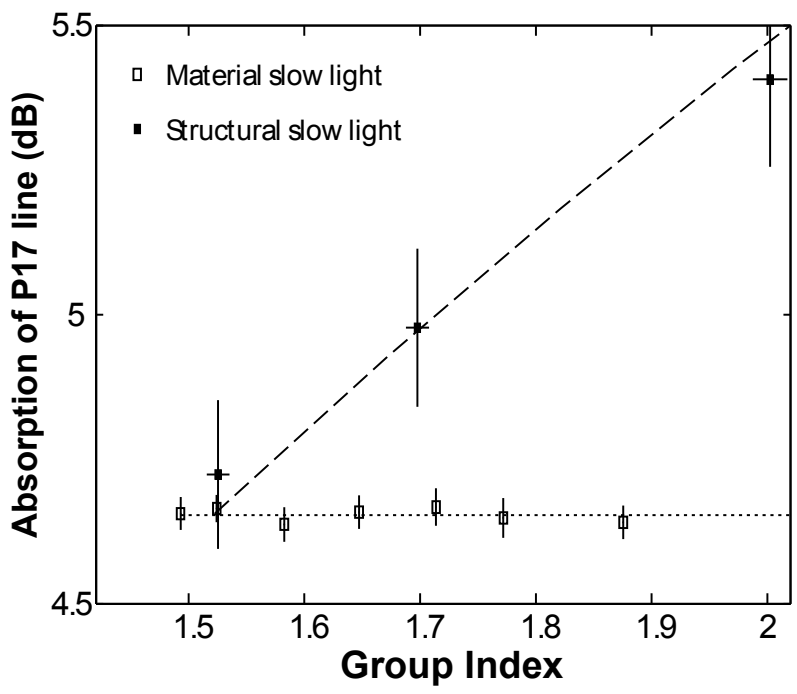

Figure 7. Dependence of the optical absorption of molecules on the group index for material slow light (white squares) and structural slow light (black squares). The dotted line represents an absorption coefficient constant with group index and the dashed line represents the theoretical model for the absorption enhancement in macroscopic cavities, predicting a linear dependence between absorption coefficient and group index for either small group indices or absorptions. Larger error bars seen for the structural slow light data points are related to normalization issues due to a non-uniform intensity build-up of the line background, whereas gain uniformity is ensured for the material slow light experiment via simultaneous tuning of the pump and signal waves.

\section{DISCUSSION}

Experimental results of two different fiber-based slow-light systems were presented in this paper. The absorption coefficient was measured for several group velocities in the very same fiber gas cell and for the same acetylene absorption lines using material and structural slow light. As illustrated in Fig. 7, no enhancement of molecular absorption was observed for material slow light whereas enhancement could be observed for structural slow light. Since the dependence of the optical absorption of molecules on the group velocity is radically different depending on the slowlight generation mechanism, this clarifies that the group velocity is not the appropriate universal quantity scaling lightmatter interactions. In a periodic, lossless media such as ring resonators the velocity of energy propagation is identical to the group velocity of a quasi-monochromatic wave $\mathrm{e}^{14-18}[8,9]$. Reducing the electromagnetic energy velocity through structural slow light effectively increases the electromagnetic energy density and yields the observed enhancement of light-matter interactions. However in the presence of significant attenuation or gain part of the energy is dissipated or stored in material excitation and cannot contribute to enhance the EM energy density. The average velocity of the EM energy flow is negligibly changed by material resonances and cannot enhance the molecular absorption effect, as experimentally verified. Consequently, these experimental results suggest that the optical absorption of molecules essentially given by the coupling between the electric field of the optical wave and the electric dipole induced by the bound orbiting electrons in the molecules - can be changed only in systems where the amplitude of the electric field of the optical wave is actually enhanced when compared to a normal propagation ${ }^{19}$. The fact that the electric field of light is denser decisively rules over pure signal velocity changes.

\section{ACKNOLEDGEMENTS}

We acknowledge the support from the Swiss National Science Foundation through projects 200020-121860 and 200021134546 and from the European Community's Seventh Framework Program [FP7/2007-2013] under grant agreement $\mathrm{n}^{\circ} 219299$ (GOSPEL project). This study was also realized in the framework of the European COST Action 299 "FIDES". 


\section{REFERENCES}

[1] Khurgin, J. B. and Tucker, R. S., Slow light: Science and applications CRC Press, Taylor and Francis Group, Boca Raton (2009).

[2] Soljacic, M., Johnson, S. G., Fan, S. H., Ibanescu, M., Ippen, E. and Joannopoulos, J. D., "Photonic-crystal slowlight enhancement of nonlinear phase sensitivity," Journal of the Optical Society of America B-Optical Physics 19(9), 2052-2059 (2002)

[3] Monat, C., Corcoran, B., Ebnali-Heidari, M., Grillet, C., Eggleton, B. J., White, T. P., O'Faolain, L. and Krauss, T. F., "Slow light enhancement of nonlinear effects in silicon engineered photonic crystal waveguides," Opt. Express 17(4), 2944-2953 (2009)

[4] Shi, Z. M., Boyd, R. W., Gauthier, D. J. and Dudley, C. C., "Enhancing the spectral sensitivity of interferometers using slow-light media," Optics Letters 32(8), 915-917 (2007)

[5] Terrel, M., Digonnet, M. J. F. and Fan, S., "Performance comparison of slow-light coupled-resonator optical gyroscopes," Laser \& Photonics Reviews 3(5), 452-465 (2009)

[6] Wen, H., Terrel, M., Fan, S. and Digonnet, M., "Sensing With Slow Light in Fiber Bragg Gratings," Sensors Journal, IEEE 12(1), 156-163 (2012)

[7] Mortensen, N. A. and Xiao, S., "Slow-light enhancement of Beer-Lambert-Bouguer absorption," Applied Physics Letters 90(14), 141108-141103 (2007)

[8] Jensen, K. H., Alam, M. N., Scherer, B., Lambrecht, A. and Mortensen, N. A., "Slow-light enhanced light-matter interactions with applications to gas sensing," Optics Communications 281(21), 5335-5339 (2008)

[9] Hochman, A. and Leviatan, Y., "Efficient and spurious-free integral-equation-based optical waveguide mode solver," Opt. Express 15(22), 14431-14453 (2007)

[10] Chin, S., Dicaire, I., Beugnot, J.-C., Foaleng-Mafang, S., Gonzalez-Herraez, M. and Thévenaz, L., "Material Slow Light Does Not Enhance Beer-Lambert Absorption," in Slow and Fast Light (SL) 2009, p. SMA3, Optical Society of America, Honolulu, Hawaii (2009).

[11] Thevenaz, L., "Slow and fast light in optical fibres," Nat Photon 2(8), 474-481 (2008)

[12] Henningsen, J., Hald, J. and Peterson, J. C., "Saturated absorption in acetylene and hydrogen cyanide in hollowcore photonic bandgap fibers," Opt. Express 13(26), 10475-10482 (2005)

[13] Dicaire, I., Chin, S. and Thévenaz, L., "Structural slow light can enhance Beer-Lambert absorption," in Slow and Fast Light (SL), p. SLWC2, Optical Society of America, Toronto, Canada (2011).

[14] Schulz-DuBois, E. O., "Energy transport velocity of electromagnetic propagation in dispersive media," Proceedings of the IEEE 57(10), 1748-1757 (1969)

[15] Yeh, P., "Electromagnetic propagation in birefringent layered media," J. Opt. Soc. Am. 69(5), 742-756 (1979)

[16] Loudon, R., "The propagation of electromagnetic energy through an absorbing dielectric," Journal of Physics A: General Physics 3(3), 233 (1970)

[17] Yariv, A., Xu, Y., Lee, R. K. and Scherer, A., "Coupled-resonator optical waveguide: a proposal and analysis," Opt. Lett. 24(11), 711-713 (1999)

[18] Santagiustina, M., "Electromagnetic Energy Velocity in Slow Light," in Slow and Fast Light (SL), p. SLTuB5, Optical Society of America, Toronto, Canada (2011).

[19] Boyd, R. W., "Material slow light and structural slow light: similarities and differences for nonlinear optics [Invited]," J. Opt. Soc. Am. B 28(12), A38-A44 (2011) 